\title{
Toma de decisión compartida y tratamiento antipsicótico: adaptación transcultural de la escala COMRADE.
}

Shared decision making and antipsychotic treatment: Transcultural adaptation of the COMRADE scale.

\author{
Ángela Gutiérrez Gea ${ }^{\text {a }}$, María Morano Guillén ${ }^{\text {b }}$, Ignacio Lara Ruiz-Granados c , José M. Villa- \\ grán Moreno d, Francisco González Saiz ${ }^{\mathrm{e}}$. \\ ${ }^{a y b}$ Psicóloga clínica. ${ }^{c, d y e}$ Psiquiatra. ${ }^{a, b, c, d y e}$ Unidad de Gestión Clínica de Salud Mental. Hospital Jerez \\ de la Frontera. Cádiz, España.
}

Correspondencia: Ángela Gutiérrez Gea (psicologangela@gmail.com)

Recibido: 24/11/2014; aceptado: 01/07/2015

\begin{abstract}
RESUMEN: Uno de los elementos característicos del modelo de salud mental comunitaria es la orientación de las intervenciones hacia la recuperación funcional del paciente con trastorno mental grave. La escala COMRADE es un instrumento que evalúa los distintos aspectos de la comunicación interpersonal en el momento de la interacción del clínico con el usuario. El objetivo de este trabajo es presentar el proceso de traducción y adaptación a lengua española de la escala COMRADE en una población de pacientes psicóticos, así como explorar la comprensibilidad del mismo por parte de sus destinatarios. Para la elaboración de la versión española preliminar empleamos un procedimiento de "traducción en grupo" y "traducción-retrotraducción". Para la verificación de la aplicabilidad transcultural de la COMRADE utilizamos una de las denominadas "técnicas inespecíficas", en concreto la denominada "estudio piloto" ("field pretest"). Para ello se realizó un muestreo de conveniencia de 35 pacientes con trastornos del espectro psicótico procedentes de distintos dispositivos de salud mental de la Unidad de Gestión Clínica de Salud Mental del Área de Gestión Sanitaria Norte de Cádiz. La mayoría de los ítems obtienen porcentajes altos o muy altos en la categoría A, correspondiente al máximo nivel de comprensión, siendo mínimo el observado en la categoría C. En función de ello se realizaron ajustes que permitieron configurar la versión definitiva de esta escala. Pensamos que la escala COMRADE será una herramienta útil en el trabajo con estos pacientes para una toma de decisión compartida sobre sus tratamientos. PALABRAS CLAVE: toma de decisiones, evaluación, trastorno mental grave, tratamiento.
\end{abstract}

ABSTRACT: Functional recovery for patients with severe mental disorder is one main feature of community mental health. COMRADE scale is an assessing tool for different aspects about interpersonal communication between clinicians and users. Introducing the process followed for adapting and translating (from English to Spanish language) this scale is the main objective of this paper, as much as exploring its comprehension. We chose a procedure of "group translation" and "translation-backtranslation" for elaborating the preliminary Spanish version. "Field pretest" was the specific technique we used for the verification of COMRADE transcultural applicability. A sample of 35 patients with psychotic spectrum disorder was arranged throughout "The Area de Gestión Sanitaria Norte de Cádiz". Most of items obtained "high" or "very high" percentages on "A" category, (maximum level of comprehension), while those obtained in "C" category (minimum level of comprehension) were "low" or "very low". Later arrangements were done for obtaining the definitive version of the scale based on these results. Therefore, we think that the COMRADE scale will become a useful tool for shared decisions about treatments between clinicians and patients.

KEY WORDS: decision making, evaluation, severe mental disorder, treatment. 


\section{Introducción}

Uno de los elementos característicos del modelo de salud mental comunitaria es la orientación de las intervenciones hacia la recuperación funcional del paciente con trastorno mental grave. Esto supone hacerle partícipe de este proceso, desde una perspectiva de empoderamiento y normalización. Pero ello no es posible si no incorporamos al paciente en las decisiones terapéuticas y rehabilitadoras que le atañen directamente (1).

La consulta, como espacio de encuentro entre usuarios y profesionales de salud mental, constituye un acto de comunicación interpersonal que debe proporcionar información adecuada como paso previo al acto de tomar una decisión sobre el tratamiento a seguir(2). Las variables que condicionan esta interacción pueden determinar en parte la evolución clínica del caso, por lo que su estudio está siendo objeto de un creciente interés (3-4). Las barreras en la comunicación clínico-paciente van más allá de la falta de información percibida por los usuarios, estando mediadas por múltiples factores que condicionan su participación en el proceso (5). El llamado "Modelo de Toma de Decisión Compartida" se sitúa entre las limitaciones del consentimiento informado y los excesos del modelo paternalista, y se contempla cada vez con más fuerza como el adecuado para la comunicación entre clínicos y usuarios. Este modelo se ha mostrado útil en personas con problemas de salud crónicos con gran impacto en su calidad de vida y que precisan tratamiento y cuidados a largo plazo, por lo que sería especialmente aplicable a los trastornos mentales graves. A pesar de ello, aún hay escasas evidencias de su aplicabilidad y efectividad en esta población(6-7).

La evaluación de este proceso de transmisión e intercambio de información, denominado en la literatura anglosajona "comunicación de riesgos", requiere el establecimiento previo de una definición y debe plantear, además, un objetivo claro y posibilista (2). Mientras que la comunicación de riesgos puede ser definida como un intercambio abierto y bi-direccional de información y opinión sobre los beneficios y los riesgos de distintas alternativas de tratamiento, su objetivo sería la toma de decisión informada. Los instrumentos de evaluación que hasta el momento se habían aproximado a la medición de este constructo procedían del campo de la investigación sobre la toma de decisión general en el ámbito de la salud y estaban más orientados a los efectos de la toma de decisión compartida que a la evaluación del proceso en sí mismo. Adrian Edwards y cols. revisaron los principales instrumentos existentes hasta el momento, identificando los ítems más relevantes para la medida de este constructo (8), desarrollando finalmente el Combined Outcome Measure for Risk communication And treatment Decision making Effectineness (COMRADE). La escala COMRADE es un instrumento que evalúa los distintos aspectos de la comunicación interpersonal en el momento de la interacción del clínico con el usuario. Lo hace en términos de comunicación de riesgos y efectividad de la toma de decisiones, teniendo 
en cuenta los preceptos teóricos de la toma de decisión compartida. Inicialmente, esta escala de evaluación se diseñó para ser empleada en cualquier ámbito clínico, aunque su estudio de validación se realizó sobre una muestra de pacientes con fibrilación auricular, prostatismo y síntomas perimenopáusicos (2). Hasta donde nosotros sabemos, este instrumento de evaluación no ha sido empleado ni validado en muestras de pacientes psicóticos, ni existía previamente ninguna versión en lengua castellana.

Muchos de los instrumentos de medida y evaluación psicopatológica disponibles en nuestra lengua han sido desarrollados previamente en otros países. El desarrollo de versiones que puedan emplearse de manera fiable en nuestro entorno requiere seguir un proceso que asegure la equivalencia conceptual con el instrumento original, pero que también garantice su aplicabilidad transcultural (9). La adaptación de una herramienta de medida incluye no solo la traducción del mismo y su adaptación cultural e idiomática, sino también la verificación de las características psicométricas de fiabilidad y validez (9-10). En los últimos años se han producido avances importantes en este campo y se han desarrollado unas directrices internacionales consensuadas para guiar este proceso (10).

El objetivo de este trabajo es presentar el proceso de traducción y adaptación a lengua española de la escala COMRADE en una población de pacientes psicóticos, así como explorar la comprensibilidad del mismo por parte de sus destinatarios.

\section{Metodología}

Para la construcción de la versión española de la escala COMRADE seguimos el procedimiento clásico en tres fases (9) consistente en, a) realizar la traducción del instrumento, b) asegurar la adecuada adaptación idiomática y cultural y c) comprobar que cumple criterios psicométricos mínimos de fiabilidad y validez. En este trabajo presentamos los resultados del desarrollo de las dos primeras etapas, estando actualmente el instrumento en fase de validación.

La COMRADE es un instrumento cuyo propósito global es la evaluación de la percepción subjetiva del paciente sobre la calidad de la comunicación con el clínico y su participación en las decisiones sobre su tratamiento. Está diseñada como una escala autoadministrada compuesta por un total de 20 ítems que puede cumplimentarse en unos 5-10 minutos. Se compone de dos subescalas de 10 ítems cada una, que reflejan dos dimensiones factoriales; "Satisfacción con la comunicación" y "Confianza en la decisión tomada". Esta última valora la percepción del paciente sobre el grado en que se siente implicado o partícipe en las decisiones que atañen a su propio tratamiento. Las respuestas a cada ítem están codificadas mediante una escalación tipo Likert de 5 grados que oscilan en un rango que va de 1 ("Completamente en 
desacuerdo") a 5 ("Completamente de acuerdo"). El estudio original de validación arroja buenos parámetros de calidad psicométrica (2).

1. Elaboración de una versión española preliminar de la COMRADE

El primer lugar se solicitó al autor de la versión original de la COMRADE la autorización para la realización de la versión española adaptada. Este primer paso es importante, ya que no solo evita la existencia de versiones no autorizadas que puedan diferir en sus propiedades psicométricas, sino que facilita la colaboración necesaria para realizar con posterioridad la adecuada supervisión de la versión retrotraducida. A continuación empleamos un procedimiento de "traducción en grupo" y "traducción-retrotraducción" (9). En primer lugar, se procedió a una traducción por separado por parte de dos profesionales de salud mental con adecuados conocimientos del inglés (I.L.RG. y F.G.S.). Posteriormente, estas dos versiones se trabajaron en común mediante la comparación activa y el consenso por parte de estos dos profesionales, obteniendo una versión unificada que fue supervisada por otro profesional con conocimientos del inglés (J.V.M.). En la elaboración de esta versión española preliminar se aseguró la equivalencia técnica (estructura general y adecuación gramáticosintáctica), la equivalencia semántica y la equivalencia conceptual teórica (ajuste de los aspectos denotativos y connotativos de los términos). Seguidamente, la versión española fue retrotraducida al inglés por una profesional de salud mental bilingüe de origen británico (E.A.H.). Posteriormente enviamos la versión retrotraducida al autor de la COMRADE (Dr. Adrian Edwards), quien la comparó con la versión original inglesa, verificando su equivalencia conceptual.

2. Verificación de la aplicabilidad transcultural de la COMRADE

Dentro del proceso de adaptación de una escala de evaluación procedente de otro entorno, la realización de una adecuada traducción es una fase necesaria pero no suficiente, por lo que se precisa comprobar su equivalencia conceptual, cultural e idiomática. Para ello utilizamos una de las denominadas "técnicas inespecíficas", en concreto la denominada "estudio piloto" ("field pretest") (9). Consiste en aplicar la versión preliminar del instrumento a una muestra de pacientes representativa de la población con la que se va a emplear el mismo. A cada sujeto se le pregunta individualmente acerca de lo que entiende de cada uno de los ítems y se le pide que exprese sus dificultades de comprensión y/o cumplimentación, al tiempo que se compara con el concepto originalmente planteado por el autor.

Para ello se realizó un muestreo de conveniencia de 35 pacientes con trastornos del espectro psicótico procedentes de distintos dispositivos de salud mental de la Unidad de Gestión Clínica de Salud Mental del Área de Gestión Sanitaria Norte de Cádiz, que aceptaron participar voluntariamente tras ser adecuadamente informados (USMC-Sanlúcar de Barrameda, USMC-Jerez de la Frontera, Comunidad Terapéutica San Miguel, y Hogar Residencial San Mateo de Jerez de la Frontera). La muestra estuvo finalmente compuesta por 7 mujeres y 28 hombres, con un rango de edad 
comprendido entre los 20 y los 60 años. El diagnóstico más prevalente fue el de Esquizofrenia Paranoide (F20.0) (22 sujetos), siendo los restantes; Trastorno Esquizoafectivo (F25) (5 sujetos), Trastorno de Ideas Delirantes Persistentes (F22.0) (2 sujetos), Otros Trastornos Psicóticos no Orgánicos (F29) (5 sujetos) y Esquizofrenia Hebefrénica (F20.10) (1 sujeto). Casi todos los pacientes (34 sujetos) presentaron un nivel de funcionamiento general comprendido entre 21 y 40, medido mediante la Escala de Evaluación de Actividad General del DSM-IV.

La prueba fue aplicada por dos residentes PIR de último año (A.G.G. y M.M.G.) mediante una entrevista cara a cara con cada paciente en el dispositivo en que habitualmente eran atendidos. A cada sujeto se le informó del carácter voluntario de la prueba, se le aseguró la confidencialidad, se le explicó el objetivo del estudio y el propósito concreto de la escala COMRADE; conocer su opinión sobre la atención recibida, su percepción de la relación clínico-paciente y su valoración sobre el grado de implicación personal en las decisiones que se toman sobre su tratamiento antipsicótico, referidas a su psiquiatra habitual. Durante la prueba, tanto el paciente como el entrevistador contaban con sendas copias de la COMRADE. El paciente procedía a leer el enunciado de cada ítem y seguidamente se le pedía que explicase lo que había entendido sobre el mismo, pudiendo solicitar al entrevistador aclaraciones en caso de duda. El entrevistador observaba el posible grado de discrepancia entre lo que el paciente había comprendido de cada enunciado y la esencia conceptual del mismo. En función de ello, el entrevistador registraba de forma estandarizada el grado de comprensión de cada ítem en tres categorías; A) "El paciente comprende el enunciado en su totalidad, capta la esencia conceptual", B) "Comprensión parcial del enunciado, se requiere alguna aclaración" y C) "Hay una importante ausencia de comprensión o existe una comprensión errónea o se requiere una aclaración completa del mismo". De forma complementaria, se registra de forma no estandarizada impresiones o comentarios relevantes de los pacientes sobre cada enunciado.

El tiempo promedio de cumplimentación de la escala COMRADE, desde que el paciente tiene la escala en sus manos, se realiza el encuadre hasta que el paciente finaliza la escala y se rellena el apartado de comprensión es de unos 12 minutos, con grandes diferencias en función del grado de deterioro del paciente, habiéndose observado un tiempo superior en los pacientes ingresados en la comunidad terapéutica y en los dispositivos residenciales, en relación a los pacientes con seguimiento comunitario, donde el funcionamiento y el rendimiento cognitivo fue más elevado. Algunos pacientes se mostraron confusos al responder a algunos ítems, identificando erróneamente a su psiquiatra con otros profesionales sanitarios (médico de atención primaria, enfermero especialista, psicólogo clínico, etc). En numerosos casos tuvimos que recordar al paciente que el cuestionario es anónimo, ya que muchos pensaban que su médico iba a conocer las respuestas, lo que podría condicionar la veracidad de la información registrada. 
3. Elaboración de la versión española definitiva de la COMRADE

En la Tabla 1 se exponen los porcentajes de cada una de las tres categorías en que se ha codificado el grado de comprensión de cada ítem de la escala por parte de los pacientes. Como puede verse, la mayoría de los ítems obtienen porcentajes altos o muy altos en la categoría A, correspondiente al máximo nivel de comprensión, siendo mínimo el observado en la categoría C. Considerando la distribución de estas frecuencias, los ítems que presentaron mayor dificultad de comprensión fueron el 5 de la subescala "Satisfacción con la comunicación" ("5. El médico me ha explicado lo que he necesitado sobre la información que me dio de los tratamientos disponibles") y el 6 de la subescala "Confianza en la decisión tomada" ("6. Estoy satisfecho con que se me haya informado adecuadamente sobre los aspectos, cuestiones, importantes para la decisión"). En consecuencia, se realizó una nueva traducción de estos ítems siguiendo el mismo procedimiento que en la primera adaptación (en la Tabla 1 aparecen las traducciones definitivas).

Además de estas modificaciones justificadas por las categorías de comprensión, hemos revisado de nuevo la redacción de los enunciados originales de todos los ítems desde una perspectiva cualitativa. Las entrevistadoras tuvieron en consideración la reiteración de dificultades de comprensión más sutiles, llevando un registro de los ítems que presentaban alguna dificultad relativa, así como las paráfrasis que tuvieron que hacer para facilitar la compresión de la esencia de los ítems. Las modificaciones realizadas incidieron tanto en la estructura sintáctica, proponiendo frases de estructura más simple, como en la complejidad lingüística y conceptual de determinadas expresiones, buscando sinónimos de uso más común. El ítem 6 de la primera subescala y los ítems 1,7 y 9 de la segunda, permanecieron con su enunciado original.

Una vez incorporados todos estos cambios a la versión castellana de la COMRADE, se volvió a retro-traducir y se envió para su revisión a Adrian Edwards. No todos los cambios propuestos fueron aceptados por él, ya que en el proceso de retrotraducción al inglés, algunos de los cambios modificaron el significado original del ítem. Finalmente, y mediante un nuevo procedimiento de "traducción en grupo" se elaboró la versión en castellano definitiva de la COMRADE.

Por otro lado, y aunque no era un objetivo de este trabajo, en la Tabla 1 presentamos también los valores medios de las respuesta directas a cada ítem de los pacientes de la muestra, en la que pueden observarse valores relativamente altos de "satisfacción" y "confianza". 
Tabla 1.

Distribución de frecuencias de las categorías de comprensión de los ítems y puntuaciones medias de las respuestas directas.

\begin{tabular}{|c|c|c|c|c|}
\hline Escala COMRADE & $\begin{array}{c}\text { Categoría de } \\
\text { comprensión } \\
\text { A }(\%)\end{array}$ & $\begin{array}{c}\text { Categoría de } \\
\text { comprensión B } \\
(\%)\end{array}$ & $\begin{array}{c}\text { Categoría de } \\
\text { comprensión C } \\
(\%)\end{array}$ & $\begin{array}{l}\begin{array}{c}\text { Puntuación } \\
\text { media }\end{array} \\
\text { Media (D.E.) }\end{array}$ \\
\hline \multicolumn{5}{|l|}{$\begin{array}{l}\text { Subescala "Satisfacción con la } \\
\text { comunicación" }\end{array}$} \\
\hline $\begin{array}{l}\text { 1.El médico me ha informado de las distintas } \\
\text { opciones de tratamiento }\end{array}$ & 97.1 & 2.8 & 0 & $3.7(1,50)$ \\
\hline $\begin{array}{l}\text { 2.El médico me ha dejado opinar sobre las } \\
\text { distintas opciones de tratamiento }\end{array}$ & 88.5 & 11.4 & 0 & $3.4(1,42)$ \\
\hline $\begin{array}{l}\text { 3.El médico me ha dejado preguntar todo lo } \\
\text { que he necesitado sobre las distintas opciones } \\
\text { de tratamiento. }\end{array}$ & 77.1 & 22.8 & 0 & $3.4(1,59)$ \\
\hline $\begin{array}{l}\text { 4. El médico me ha dado la información que } \\
\text { he necesitado sobre las distintas opciones de } \\
\text { tratamiento. }\end{array}$ & 80 & 20 & 0 & $3.6(1,36)$ \\
\hline $\begin{array}{l}\text { 5. El médico me ha explicado lo que he } \\
\text { necesitado sobre las distintas opciones de } \\
\text { tratamiento (de las que me informó) }\end{array}$ & 51.4 & 43 & 8.5 & $3.1(1,45)$ \\
\hline $\begin{array}{l}\text { 6. La información que me han dado ha sido } \\
\text { fácil de entender. }\end{array}$ & 88.5 & 11.4 & 0 & $\begin{array}{c}3.9 \\
(1,35) \\
\end{array}$ \\
\hline $\begin{array}{l}\text { 7. Conozco las ventajas de tomar tratamiento } \\
\text { (o de no tomar tratamiento) }\end{array}$ & 88.5 & 11.4 & 0 & $4.3(1,15)$ \\
\hline $\begin{array}{l}\text { 8. Conozco las desventajas de tomar } \\
\text { tratamiento (o no tomar tratamiento) }\end{array}$ & 94.2 & 5.7 & 0 & $4.2(1,26)$ \\
\hline $\begin{array}{l}\text { 9. El médico me ha dejado decir que } \\
\text { tratamiento he pensado que era el mejor para } \\
\text { mi. }\end{array}$ & 94.2 & 2.8 & 2.8 & $3.2(1,63)$ \\
\hline $\begin{array}{l}\text { 10. El médico me ha dejado participar en } \\
\text { las decisiones que se tomaron durante la } \\
\text { consulta. }\end{array}$ & 82.7 & 14.2 & 2.8 & $3.2(1,65)$ \\
\hline \multicolumn{5}{|l|}{$\begin{array}{l}\text { Subescala "Confianza en la decisión } \\
\text { tomada" }\end{array}$} \\
\hline $\begin{array}{l}\text { 1.En general estoy satisfecho con la } \\
\text { información que se me ha dado }\end{array}$ & 97.1 & 2.8 & 0 & $4.0(1,30)$ \\
\hline $\begin{array}{l}\text { 2- Mi médico y yo estamos de acuerdo sobre } \\
\text { qué tratamiento (o no tratamiento) era el más } \\
\text { adecuado para mí. }\end{array}$ & 88,5 & 11,4 & 0 & $3.7(1,62)$ \\
\hline $\begin{array}{l}\text { 3- Puedo hablar otra vez con mi médico sobre } \\
\text { mi enfermedad fácilmente si lo necesito. }\end{array}$ & 82,8 & 17,1 & 0 & $4.3(1,08)$ \\
\hline $\begin{array}{l}\text { 4- Estoy contento con el modo en que se ha } \\
\text { tomado la decisión sobre mi tratamiento en } \\
\text { consulta. }\end{array}$ & 82,8 & 14,2 & 2,86 & $4.0(1,28)$ \\
\hline $\begin{array}{l}\text { 5- Estoy seguro de que la decisión que se } \\
\text { tomó fue la más adecuada para mi caso. }\end{array}$ & 82,8 & 14,2 & 2,86 & $4.0(1,22)$ \\
\hline $\begin{array}{l}\text { 6- Estoy contento con que se me haya } \\
\text { informado bien sobre los aspectos importantes } \\
\text { para la decisión. }\end{array}$ & 60 & 34,2 & 5,7 & $3.8(1,31)$ \\
\hline 7- Está claro cuál es la mejor opción para mí. & 80 & 17 & 2,8 & $3.5(1,66)$ \\
\hline 8-Conozco las opciones de tratamiento que tengo. & 77,1 & 22,8 & 0 & $3.6(1,35)$ \\
\hline $\begin{array}{l}\text { 9- Siento que la decisión se ha tomado } \\
\text { después de haber sido debidamente } \\
\text { informado. }\end{array}$ & 80 & 20 & 0 & $3.4(1,48)$ \\
\hline $\begin{array}{l}\text { 10- La decisión tomada sobre el tratamiento } \\
\text { tiene en cuenta las cosas que son más } \\
\text { importantes para mi. }\end{array}$ & 71,4 & 25,7 & 2,8 & $4(1,30)$ \\
\hline
\end{tabular}




\section{Discusión y conclusiones}

El objetivo fundamental de este trabajo ha sido presentar los resultados del proceso de adaptación al castellano de la escala COMRADE en pacientes psicóticos, como una etapa previa al proceso de validación de la misma, que está teniendo lugar en estos momentos. Lo presentamos de forma independiente debido a la entidad metodológica que a nuestro juicio tiene un procedimiento riguroso de estas características.

En la primera parte se realizó una versión potencialmente definitiva tras un proceso de traducción y retro-traducción ya descrito, por ser el procedimiento aceptado habitualmente para asegurar la equivalencia conceptual en la adaptación de los cuestionarios a poblaciones con un idioma y cultura distintos de la población para la que se creó la prueba original. En opinión de nuestro equipo y coincidiendo con el autor de la escala, Adrian Edwards, este objetivo se ha alcanzado satisfactoriamente. En otros estudios, para la adaptación de instrumentos, se ha procedido a reproducir las fases de creación del cuestionario original en la población objeto de investigación. Este procedimiento permite una equivalencia conceptual más alta, pero requiere más tiempo y recursos, lo que puede comprometer a la comparabilidad de los resultados (11).

En la segunda parte hemos evaluado el grado en que los pacientes comprenden adecuadamente lo que se les está preguntando. Tras cuantificar las categorías de comprensión y tras una valoración cualitativa de sus respuestas, realizamos los ajustes pertinentes que permitieron configurar la versión definitiva de esta escala. Consideramos que éste es un punto fuerte de nuestro trabajo debido a las características de los pacientes psicóticos como población diana a la que se quiere adaptar (alteración en la función ejecutiva, paranoidismo, etc.), teniendo en cuenta, además, que se trata de un instrumento de evaluación de tipo autoadministrado. Aunque la muestra no fue seleccionada de forma aleatoria, se realizó un esfuerzo en alcanzar su representatividad en términos de distribución por sexo, edad, diagnósticos dentro del espectro psicótico y dispositivo de referencia, por lo que, consecuentemente, hay variabilidad en grados de deterioro, funcionalidad y años de evolución de la enfermedad.

Pensamos que la escala COMRADE será una herramienta útil en el trabajo con estos pacientes para una toma de decisión compartida sobre sus tratamientos, desde el paradigma del empoderamiento.

\section{BIBLIOGRAFÍA}

(1) Villagrán, JM, Lara Ruiz-Granados I, González-Saiz, F. El proceso de decisión compartida en el tratamiento del paciente psiquiátrico: estudios empíricos y evaluación de la capacidad. Rev. Asoc. Esp. Neuropsiq 2014; 34: 491-506.

(2) Edwards A, Elwyn G, Hood K, Robling M, Atwell C, Holmes-Rovner M, et al. The development of COMRADE--a patient-based outcome measure to evaluate the effectiveness of 
risk communication and treatment decision making in consultations. Patient Educ Couns. 2003 Jul;50(3):311-22.

(3) Power TE, Swartzman LC, Robinson JW. Cognitive-emotional decision making (CEDM): a framework of patient medical decision making. Patient Educ Couns. 2011 May;83(2):163-9.

(4) A practitioner's guide to interpersonal communication theory: An overview and exploration of selected theories Carma L. Bylund, Emily B. Peterson, Kenzie A. Cameron Patient Educ Couns. Author manuscript; available in PMC 2013 June 1.Published in final edited form as: Patient Educ Couns. 2012 June; 87(3): 261-267.

(5) Joseph-Williams N, Elwyn G, Edwards A. Knowledge is not power for patients: A systematic review and thematic synthesis of patient-reported barriers and facilitators to shared decision making. Patient Educ Couns. 2014 Mar;94(3):291-309

(6) Hamann J, Leucht S, Kissling W. Shared decision making in psychiatry. Acta Psychiatr Scand. 2003 Jun;107(6):403-9.

(7) Reichhart T, Kissling W, Scheuring E, Hamann J. [Patient participation in german psychiatry - a critical review]. Psychiatr Prax. 2008 Apr;35(3):111-21.

(8) Edwards A, Elwyn G, Smith C, Williams S, Thornton H. Consumers'views of quality in the consultation and their relevance to "shared decision making" approaches. Health Expect 2001; 4: 151-162.

(9) Gaite L, Ramirez N, Herrera S, Vázquez-Barquero JL. Traducción y adaptación transcultural de instrumentos de evaluación en psiquiatría: aspectos metodológicos. Archivos de Neurobiología 1997; 60: 91-111.

(10) Muñiz J, Elosua P, Hambleton RK. Directrices para la traducción y adaptación de los tests: segunda edición. Psicothema 2013, Vol. 25, No. 2, 151-157

(11) Blanco-Aparicio M, Vázquez Rodríguez, I, Verea Hernando, H. Adaptación Transcultural al español del Airways Questionnaire 20 (AQ20), un cuestionario de calidad de vida abreviado para la evaluación clínica del asma y la EPOC. Archivos de Bronconeumología. 2009; 45 (1): 24-29. 